\title{
Multiplexing curvature sensors using fibre segment interferometry for lateral vibration measurements
}

\author{
Thomas Kissinger, Edmon Chehura, Stephen W. James and Ralph P. Tatam* \\ Engineering Photonics, Cranfield University, Cranfield, MK43 0AL, United Kingdom \\ *r.p.tatam@cranfield.ac.uk
}

\begin{abstract}
Dynamic fibre-optic curvature sensing is demonstrated by interrogating chains of fibre segments, separated by broadband Bragg grating reflectors, using range-resolved interferometry (RRI). Four fibre strings, containing four fibre segments each of gauge length $20 \mathrm{~cm}$, are attached to the opposing sides of a support structure and the resulting differential strain measurements allow inference of lateral displacements of a cantilever test object. Dynamic tip displacement resolutions in the micrometre range at an interferometric bandwidth of $21 \mathrm{kHz}$ demonstrate the suitability of this approach for highly sensitive and cost-effective fibre-optic directional vibration measurements of smart structures.
\end{abstract}

Keywords: Range-Resolved Interferometry, Shape Sensing, Curvature Sensing, Fibre Segment Interferometry

\section{INTRODUCTION}

Fibre optic shape sensing [1-4] (FOSS), sometimes referred to as bend or curvature sensing, allows the curvature, and therefore the curvature of the structure to which the fibre or fibre arrangement is attached, to be followed through space and permits the inference of lateral displacements directly from the curvature measurements. FOSS techniques are generally based on evaluating the differential strain that results from the curvature of a fibre/fibre arrangement. Prior infibre grating based approaches have used fibre Bragg gratings (FBG) [1] or long-period gratings [2]. Quasi-distributed FBG-based approaches, where a large number of FBG sensors are interrogated using frequency domain reflectometers, have also become popular [3], in areas such as structural health monitoring and medical endoscope tracking, mainly due to their capability for high density deployment. Interferometric approaches [4] have also been investigated for FOSS, but, while potentially offering fast and highly sensitive measurements, practical applications appear to have suffered from difficulties in multiplexing sensors. The approach proposed in this paper employs fibre segment interferometry (FSI) [5], which is a promising technique because it allows the multiplexing of many interferometric sensor segments using range-resolved interferometry (RRI) [6], where the RRI is designed to exploit the potential that robust and costeffective laser diodes originating in the telecoms industry offer to sensing applications.

Importantly, curvature sensitivity scales proportionally with the lateral fibre core distance [4] in FOSS, therefore a fibre arrangement mounted onto a flexible support structure that can follow the shape of the object under test, with lateral fibre core spacing of typically several millimetres, offers orders of magnitude increased curvature sensitivity compared to previous implementations using multicore fibres [4] with a lateral core spacing of typically $50 \mu \mathrm{m}$. In this paper, the optical setup is introduced and the details of the sensor arrangement provided, before evaluating the two-dimensional tip displacement sensitivities of the device using a cantilever test object. Finally, measurements of the cantilever tip vibrations are shown, demonstrating the suitability of this approach for directional vibration measurements.

\section{EXPERIMENT}

In this work, we use a fibre arrangement comprising of four regular single-mode fibres, where each fibre is adhered to one of the four opposing sides of a flexible Polytetrafluoroethylene (PTFE, Teflon ${ }^{\mathrm{TM}}$ ) support structure. The experimental configuration and the properties of the PTFE sensing rod are illustrated in Fig. 1. In the optical setup shown in Fig. 1(a), the output from the DFB laser diode, at a wavelength of $1549 \mathrm{~nm}$, leaving the RRI interrogation unit $[5,6]$, is split by a $4 \times 1$ coupler into the four optical fibre strings (A to D) to be interrogated. The strings each include a fibre delay line, with sequential fibre length increments of several metres, with the aim of moving undesired signals due to the interference of sources from the different strings well out of the detection range. In each string, four $20 \mathrm{~cm}$ long fibre segments are created between five FBG reflectors. The desired interference signals are then generated by the interference

\footnotetext{
25th International Conference on Optical Fiber Sensors, edited by Youngjoo Chung, Wei Jin,

Byoungho Lee, John Canning, Kentaro Nakamura, Libo Yuan, Proc. of SPIE Vol. 10323,

$1032317 \cdot$ (C) 2017 SPIE · CCC code: 0277-786X/17/\$18 - doi: 10.1117/12.2264781

Please refer to any applicable publisher terms of use.

Proc. of SPIE Vol. 10323 1032317-1
} 
of the cleaved fibre tip reflection with the FBG reflections, where the unique offset of 4, 8, 12 or $16 \mathrm{~cm}$ in each string between the cleaved fibre tip and the FBGs ensures that every signal corresponds to a unique evaluation range. In addition, undesired interference signals due to mutual interference of FBG reflections will correspond to a range multiple of $20 \mathrm{~cm}$, where no desired signals are located. In FSI, broadband FBG reflectors are used to ensure light is reflected under all conceivable temperature and strain conditions. Because the evaluated light has to pass the $4 \times 1$ coupler twice, there is power loss of a factor of 16 compared to our previous FSI implementation [5] in a single string. Therefore, the reflectivities of the broadband FBGs need to be designed to yield sufficient return light. Using a slit of width of $0.2 \mathrm{~mm}$ positioned between the fibre and the phase mask [7], FBGs of $\sim 5 \mathrm{~nm}$ spectral width (full-width half-maximum) and typical reflectivities of 200 parts per million (ppm) have been fabricated in photosensitive Fibercore PS1250/1500 fibre using a frequency-quadrupled YAG laser operating at $266 \mathrm{~nm}$.
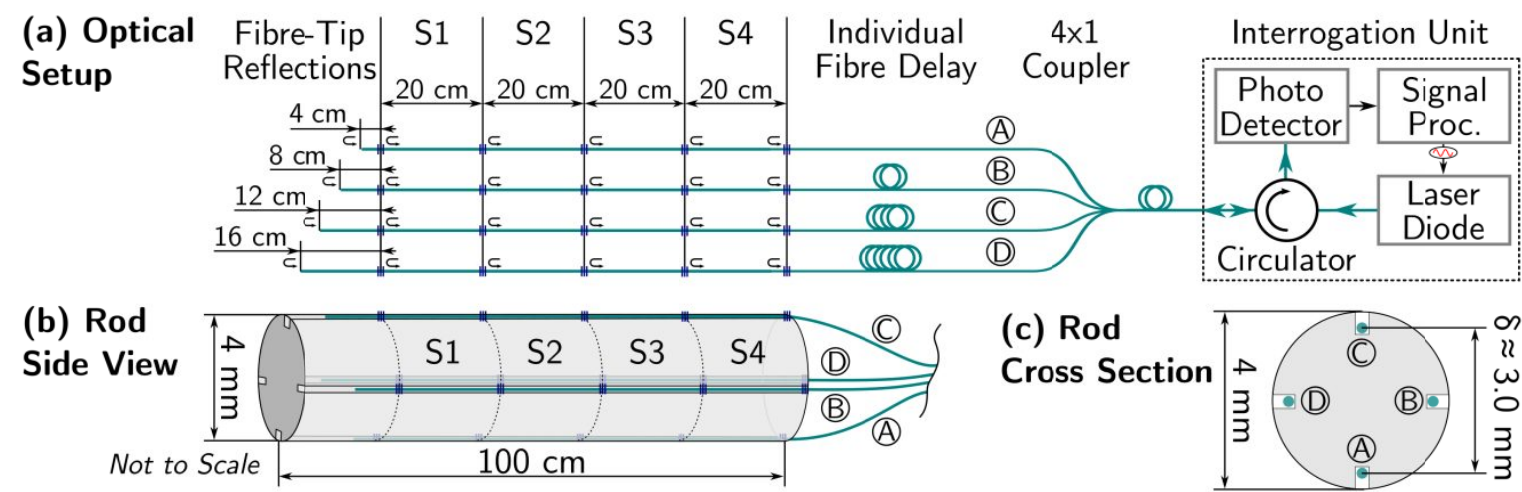

Figure 1. The fibre optical setup used is shown in (a) where 20 weak, broadband FBGs written at prescribed locations from the cleaved fibre tip in four fibre strings (A to D) form a total of 16 co-located fibre segments that correspond to four curvature sensing sections S1 to S4. Light exiting the interrogation unit is guided via a $4 \times 1$ coupler to the fibre strings and back, with an individual fibre delay for each of the four strings. In (b) the fibre mounting in channels on the opposing sides of a PTFE rod is illustrated, while the rod cross-section is drawn in (c), with the relevant fibre core lateral spacing $\delta$ marked.

Fig. 1(b) shows how the segments are co-located on the four opposing sides of the PTFE sensor rod of $4 \mathrm{~mm}$ diameter. Here, channels were cut manually in the PTFE rod to securely locate the fibre on the rod, which is illustrated in Fig. 1(c). The channels are approximately $0.6 \mathrm{~mm}$ deep, resulting in a nominal lateral fibre core spacing $\delta$ of $3.0 \mathrm{~mm}$. As this was a manual process, control of the channel depth was limited, which results in an uncertainty for the value of $\delta$. The fibres were attached to the rod by filling the channels with a cyanoacrylate adhesive. The measured differential strain signals were obtained by subtraction of the signals from the interferometers formed in strings $\mathrm{A}$ and $\mathrm{C}$ for the vertical direction and from those in strings $\mathrm{B}$ and $\mathrm{D}$ for the horizontal direction.

The physical mounting of the sensor rod onto the cantilever under test, a $15 \times 15 \mathrm{~mm}$ square bar of 316 stainless steel, is shown in Fig. 2. Here the relevant cantilever length $\mathrm{L}$ is $80 \mathrm{~cm}$ and the tip of the cantilever can be moved using a manual stage that can be oriented vertically and horizontally. In interferometric curvature sensing, the fibre needs to be attached to the structure under test only at the end points of the interferometer [4]. Furthermore, no actual strain transfer to the structure under test is needed as the differential strain measurement takes place wholly within the sensor rod. Therefore the sensor rod was fixed to the cantilever using 3M 431 aluminium tape at the locations of the FBGs only.

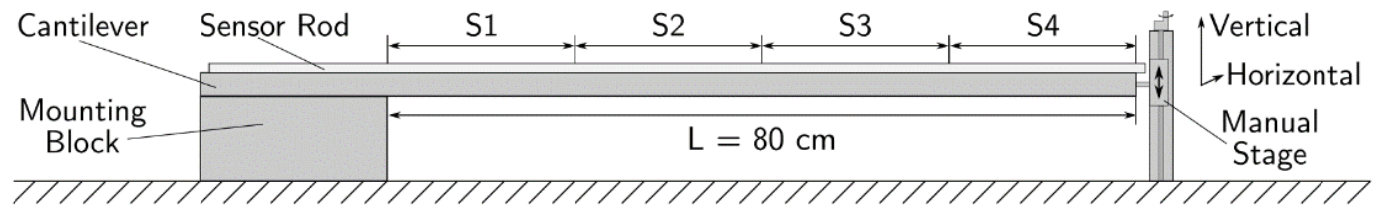

Figure 2. The physical mounting of the sensor rod on top of a square-profile stainless steel cantilever is shown. A manual stage can be used to impart controlled movements in either vertical (shown) or horizontal directions.

When the cantilever length is equal to the interferometer length, the tip displacement sensitivity $\Delta \varphi \cdot d^{-1}$, relating the measured phase difference $\Delta \varphi$ due to differential strain to the cantilever tip displacement $d$, is given by [4]:

$$
\frac{\Delta \varphi}{d}=2 \cdot 3.42 \frac{\pi \delta}{\lambda L}
$$


Here, $\delta$ is the lateral fibre core displacement, $\lambda$ is the free space emission wavelength of the laser and $L$ is the cantilever length. This assumes negligible dependence of the fibre strain sensitivity on the interrogation wavelength. The additional factor of two, compared to [4], is because FSI works in reflection, where the light passes the fibre sensor section twice.

\section{RESULTS \& DISCUSSION}

Figure 3 shows measurement results for the controlled movement of the translation stage. Figs. 3(a) and (b) show the differential strain phase responses to the horizontal and vertical movement, respectively, where in each case only the horizontal or vertical differential strain signals, respectively, are shown. The measured angular change is strongest near the cantilever start and weakest near the tip, hence the strongest signals can be seen for section S1, as qualitatively expected from mechanical theory [8]. In Fig. 3(b), the signal of section S4 shows irregular behaviour. It is suspected that this is because the final reflector is situated at or very slightly beyond the cantilever tip, causing the rod bending to deviate from the cantilever bending. Improved placement in future implementations should prevent this problem. By comparing Figs. 3(a) and (b) for the other sections (S1 to S3) it can, however, be seen that the sensor has similar sensitivities for both horizontal and vertical movements. Figs. 3(c) and (d) show the sum of the signals from all four sections for horizontal and vertical stage movements, respectively, which can be used to predict the cantilever tip displacement. Comparisons of the horizontal and vertical sensor responses in Figs. 3(c) and (d) allows an assessment of the misalignment between the sensor rod axis and the cantilever axis, revealing a small misalignment. A linear regression fit plotted in Fig. 3(c) allows determination of the tip displacement sensitivity $\Delta \varphi \cdot d^{-1}$, which was found to be $49.3 \mathrm{rad} \cdot \mathrm{mm}^{-1}$, with a similar value expected for the vertical movement if the irregularity in section $\mathrm{S} 4$ was not present. The theoretical value calculated using Eq. (1) for $\delta=3.0 \mathrm{~mm}$ is $52.0 \mathrm{rad} \cdot \mathrm{mm}^{-1}$. The $5 \%$ deviation could be explained by discrepancies in the lateral fibre distance $\delta$ or other non-idealities, such as imperfect strain transfer between the fibres and the PTFE rod. It is anticipated that better manufacture and control of the shape of the support structure should improve this.
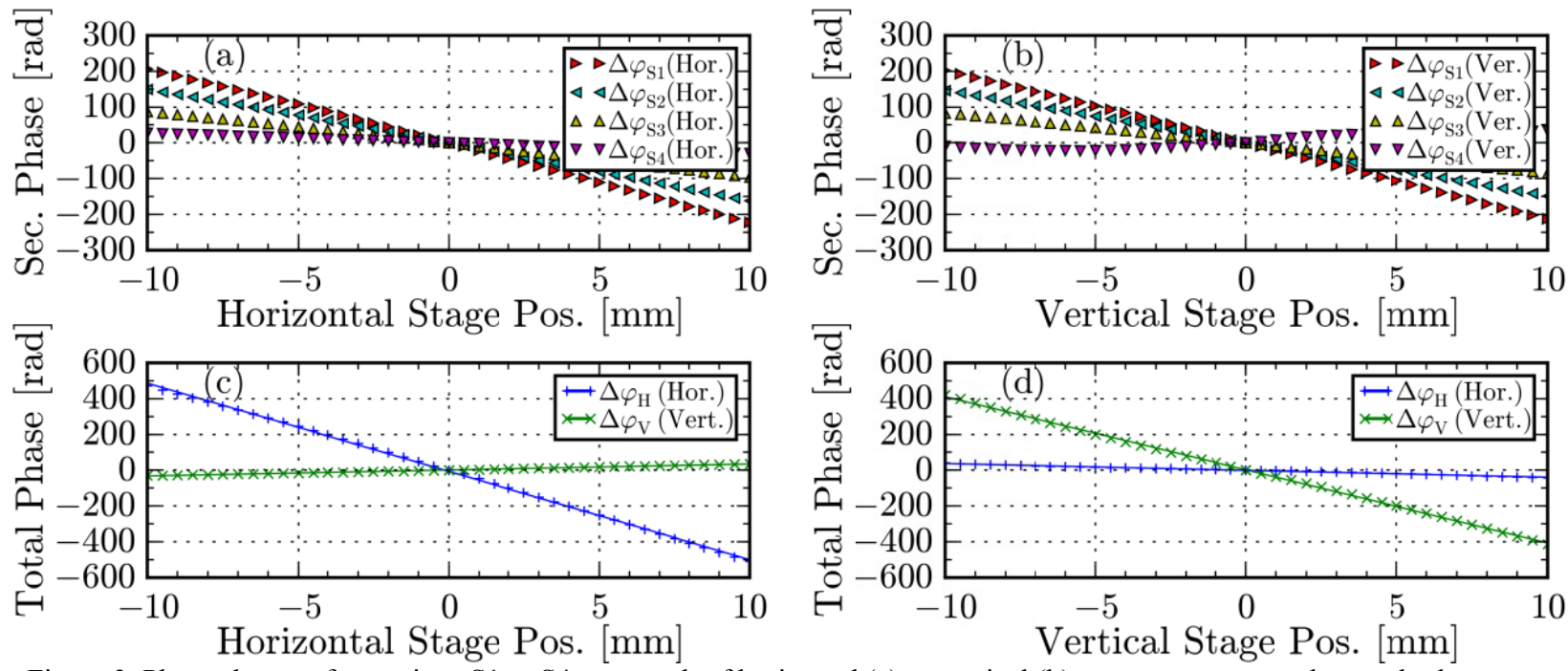

Figure 3. Phase changes for sections S1 to S4 as a result of horizontal (a) or vertical (b) stage movement, where only the components in the stage direction are plotted for clarity. For the same measurement, (c) and (d) show the total phase change, summed up over sections S1 to S4, now showing both vertical and horizontal components, as a result of horizontal (c) or vertical (d) stage movement, along with linear regression fits.

Finally, the capability of this approach for lateral, directional vibration measurements is illustrated in Fig. 4, where the mechanical stage in Fig. 2 was removed to allow free vibrations. Here, the vibration direction was fixed by using a metal guide to force the cantilever to vibrate along a particular direction. Fig. 4(a) shows the response when the cantilever vibrates along the horizontal direction, while Fig. 4(b) shows the response to a vertical vibration and Fig. 4(c) shows the response to a vibration at an angle of $\approx 45^{\circ}$. In all cases, the insets, where the horizontal and vertical signals are plotted against each other, show that the vibration direction can be determined by this measurement. In all plots, the theoretical tip displacement for the nominal value of $\delta=3.0 \mathrm{~mm}$, calculated using Eq. (1), is also plotted on the secondary y-axis. This illustrates the high tip displacement resolution that can be achieved using this approach, where the instantaneous differential strain noise standard deviation can be found to be $\approx 0.2 \mathrm{rad}$ over the interferometric bandwidth of $21 \mathrm{kHz}$ 
that was used for this measurement, equating to $\approx 4 \mu \mathrm{m}$ tip displacement noise for a cantilever of $L=0.8 \mathrm{~m}$ using the theoretical tip displacement sensitivity calculated previously.
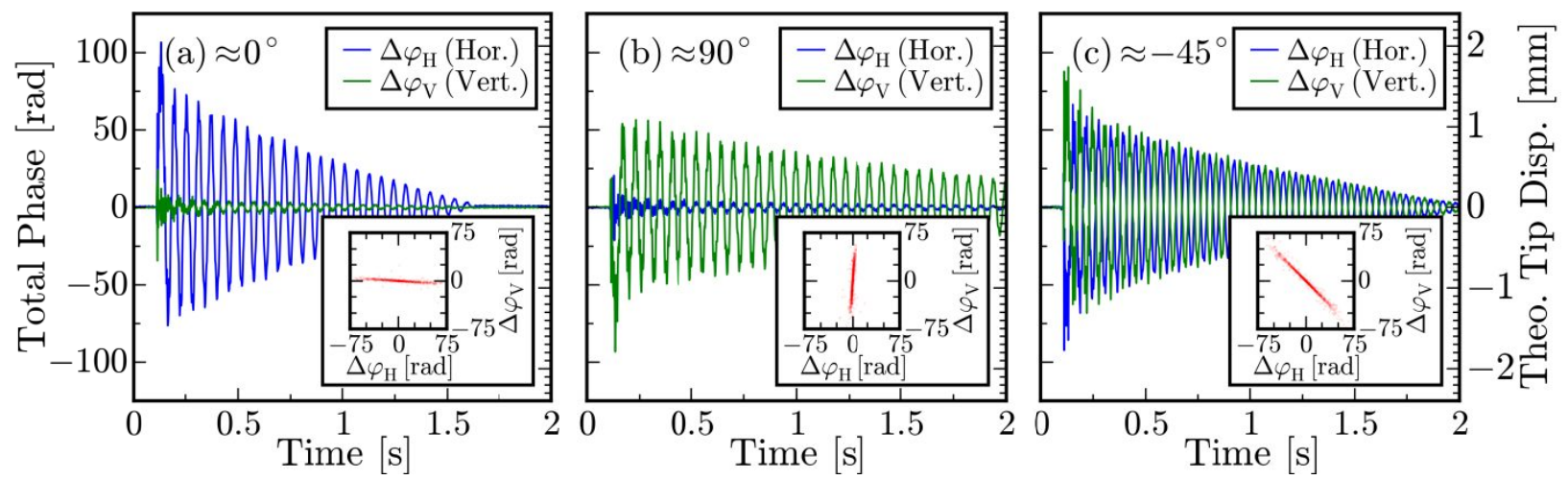

Figure 4. Three typical vibration events at the cantilever tip where the vibration direction was forced along the horizontal (a), vertical (b) or along the bisecting direction (c) are plotted over 2 s. In each plot, the primary y-axis shows the measured total phase, summed over all sections S1 to S4, while the secondary axis shows the theoretical tip displacement calculated from Eq. (1) for the nominal value of $\delta=3.0 \mathrm{~mm}$. Additionally, the insets plot the horizontal and vertical phase changes against each other to allow the determination of the vibration direction.

\section{CONCLUSION}

Fibre segment interferometry (FSI), a novel fibre sensing technique based on range-resolved interferometry (RRI), using only cost-effective and robust hardware originating from the telecom industry, has been applied to the fibre optic curvature sensing. By multiplexing a total of 16 fibre segments, four curvature sensing sections, adhered to a flexible, plastic support structure have been interrogated simultaneously. For a cantilever test object of length $0.8 \mathrm{~m}$, tip displacement resolutions of $\approx 4 \mu \mathrm{m}$ over the interferometric bandwidth of $21 \mathrm{kHz}$ have been demonstrated, illustrating the potential of the this approach for lateral, directional vibration measurements. Dynamic FOSS using FSI could in principle also be achieved using multi-core fibre, where multi-core fibres offer low weight and protrusion profile and could be embedded in composites, which might be key consideration in some applications.

\section{ACKNOWLEDGEMENTS}

The authors acknowledge the support of the Engineering and Physical Sciences Research Council (EPSRC) UK, via grant EP/N002520/1 and the InnovateUK/Aerospace Technology Institute (ATI) project BladeSense.

\section{REFERENCES}

[1] Gander, M. J., MacPherson, W. N., McBride, R., Jones, J. D. C., Zhang, L., Bennion, I., Blanchard, P. M., Burnett, J. G. and Greenaway, A. H., "Bend measurement using Bragg gratings in multicore fibre," Electron. Lett. 36(2), 120-121 (2000).

[2] Allsop, T., Gillooly, A., Mezentsev, V., Earthgrowl-Gould, T., Neal, R., Webb, D. J. and Bennion, I., "Bending and orientational characteristics of long period gratings written in D-shaped optical fiber [directional bend sensors]," IEEE Trans. Instrum. Meas. 53(1), 130-135 (2004).

[3] Duncan, R. G., Froggatt, M. E., Kreger, S. T., Seeley, R. J., Gifford, D. K., Sang, A. K. and Wolfe, M. S., "High-accuracy fiber-optic shape sensing," Proc. SPIE 6530, 1S-1S-11 (2007).

[4] Blanchard, P. M., Burnett, J. G., Erry, G. R. G., Greenaway, A. H., Harrison, P., Mangan, B., Knight, J. C., Russell, P. S. J., Gander, M. J., McBride, R. and Jones, J. D. C., "Two-dimensional bend sensing with a single, multi-core optical fibre," Smart Mater. Struct. 9(2), 132-140 (2000).

[5] Kissinger, T., Correia, R., Charrett, T. O. H., James, S. W. and Tatam, R. P., "Fiber Segment Interferometry for Dynamic Strain Measurements," J. Lightwave Technol. 34(19), 4620-4626 (2016).

[6] Kissinger, T., Charrett, T. O. H. and Tatam, R. P., "Range-resolved interferometric signal processing using sinusoidal optical frequency modulation," Opt. Express 23(7), 9415-9431 (2015).

[7] Wang, Z., Shen, F., Song, L., Wang, X. and Wang, A., "Multiplexed fiber Fabry-Perot interferometer sensors based on ultrashort Bragg gratings," IEEE Photon. Technol. Lett. 19(8), 622-624 (2007).

[8] Gere, J. M. and Timoshenko, S. P., [Mechanics of Materials (Fourth SI edition)], Nelson Thornes Ltd (1999). 
2017-04-23

\section{Multiplexing curvature sensors using \\ fibre segment interferometry for lateral vibration measurements}

\section{Kissinger, Thomas}

SPIE - International Society for Optics and Photonics

Thomas Kissinger, Edmon Chehura, Stephen W. James, Ralph P. Tatam. Multiplexing curvature sensors using fibre segment interferometry for lateral vibration measurements. 25th International Conference on Optical Fiber Sensors, 24-28 April 2017, Jeju, Republic of Korea http://dx.doi.org/10.1117/12.2264781

Downloaded from Cranfield Library Services E-Repository 